\title{
When inordinate tissue growth is beneficial: Improving silk production by increasing silk gland size
}

\author{
Xavier Bellés ${ }^{1}$ \\ 'Institute of Evolutionary Biology (CSIC-UPF), Passeig Maritim 37, 08003 Barcelona, Spain \\ Cell Research (2011) 21:862-863. doi:10.1038/cr.2011.68; published online 12 April 2011
}

Some 5000 years ago, the legendary Chinese emperor Huang-Ti ordered his wife, Hsi-Ling-Shi, to find out what was damaging his mulberry trees. HsiLing-Shi found white worms eating the mulberry leaves and spinning shiny cocoons. She accidentally dropped a cocoon into her tea cup and saw a delicate, slender thread unwind itself from the cocoon. The young princess had discovered silk $[1,2]$.

As with many agricultural crops and livestock, the silkworm (Bombyx mori) has acquired the ability to produce large amounts of silk proteins as a result of selective breeding over more than 4000 years. Silk, with a production volume of some 70000 tonnes per year [3], is mainly used in the textile industry and represents a large-scale business worldwide. The increasing demand has driven notable efforts to improve silk production through hybrid breeding, a strategy that has been very successful during the past century [3], but silk production has reached the long-expected plateau, which is consubstantial to the empirical breeding approach. However, recent work by Sheng Li and associates [4] has helped to overcome this sericultural bottleneck.

The silk gland of the silkworm is divided into three morphologically and

Correspondence: Xavier Bellés

E-mail: xavier.belles@ibe.upf-csic.es functionally distinct regions, known as posterior, middle and anterior silk gland [5]. The main constituent of silk is a protein known as fibroin, which is found in the core of the silk thread and is synthesized by the posterior silk gland. This fibroin core is covered with a layer of protein called sericin, which is produced in the middle silk gland $[3,5]$. The weight contents of fibroin and sericin in total silk proteins are approximately $75 \%$ and $25 \%$, respectively. The strategy planned by Sheng $\mathrm{Li}$ and his team [4] was to generate transgenic silkworms with increased Ras activity in the posterior silk gland in order to obtain silkworms with this part of the gland enlarged and thus able to produce more fibroin. Indeed, this sort of biotechnological brute-force approach gave the desired results.

Ras oncogenes are well-known to be key players in tumorigenesis. Amongst other effects, the activation of Ras signaling causes cell growth, whereas dysregulation can ultimately lead to oncogenesis and cancer [6]. Indeed, expression of activated Ras specifically in the larval prothoracic gland in the fruit fly (Drosophila melanogaster) increased gland size and resulted in a precocious release of molting hormone [7]. In this contribution, Philip Caldwell and colleagues used the conventional Drosophila Gal4/UAS system to modulate Ras signaling specifically in the prothoracic gland cells [7]. In the case of $B$. mori, which had been reluctant to genetic transformation for long time, an equivalent system for germline transgenesis using a piggyBack transposon-derived vector was developed in 2000 [8]. It was successfully used, for example, by Takahiro Shiotsuki and associates to study the regulatory role of juvenile hormone $(\mathrm{JH})$ esterase on the degradation of $\mathrm{JH}$ in B. mori [9]. The results were spectacular: overexpression of $\mathrm{JH}$ esterase from the embryo stage resulted in steady degradation of $\mathrm{JH}$ and, as a consequence, in larval-pupal metamorphosis after the third larval instar, two instars earlier than in wildtype silkworms [9].

Li's team [4] used the same Bombyx binary Gal4/UAS system to overexpress Ras $1^{C A}$ oncogene in the posterior silk gland, and found that size of this gland region increased by almost $60 \%$ in transgenic silkworms with respect to controls, whereas silk production also increased by approximately $60 \%$. As expected, food consumption also augmented, but only by $20 \%$, thus resulting in an estimated $30 \%$ improvement in the conversion efficiency of food to silk. Subsequently, researchers embarked on an intensive study of the mechanisms underlying the observed phenotype (Figure 1) [4]. They showed that $R a$ $s I^{C A}$ overexpression in the posterior silk gland of transgenic silkworm increased Ras activity and stimulated the phosphorylation of Raf and PI3K110, 


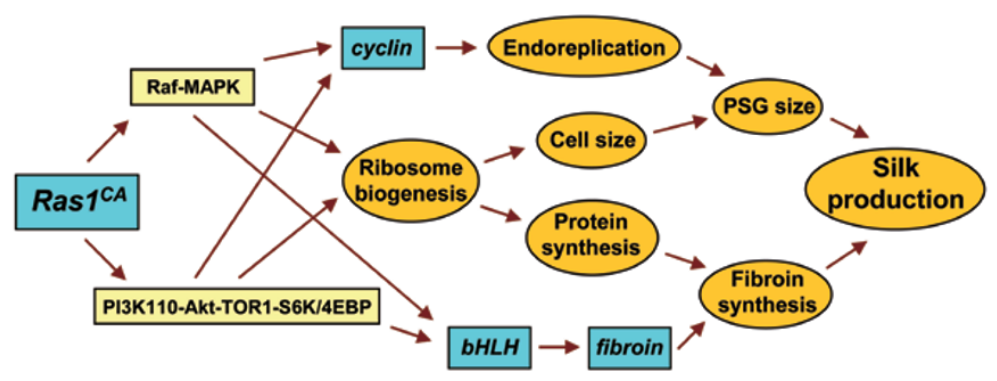

Figure 1 Basic mechanisms underlying the effect of overexpressing the oncogene Ras $1^{C A}$ in the silworm posterior silk gland (PSK) on silk yield increase.

which in turn activated the Raf-MAPK and PI3K-Akt-TORC1-S6K/4EBP pathways. Subsequently, these pathways up-regulated the cyclins involved in endoreplication, thus promoting endoreplicative cycles that increased DNA content and contributed to the size increase of the posterior silk gland. In parallel, as a consequence of activation of the PI3K-Akt-TORC1-S6K/4EBP pathways, ribosome biogenesis and protein synthesis were enhanced. Finally, Ras activation up-regulated bHLH, a transcription factor that is specifically expressed in the silk gland and enhances fibroin gene expression, resulting in a higher fibroin production and silk yield (Figure 1) [4].

In summary, a case of tissue hypertrophy induction that produced beneficial results. Indeed, as the posterior silk gland, where the overexpression of $\operatorname{Ras} 1^{C A}$ oncogene was driven, specializes in the production of fibroin, the overproduction of this protein can explain the resulting increase in silk production. A question then arises con- cerning the quality of the silk obtained, especially as the relative proportion of sericin, which is produced by the middle silk gland and forms the external silk layer, decreases. This potential silk-quality problem, however, could also be solved using a biotechnological approach. Moreover, the B. mori specimens used in the experiments were from the laboratory strain Dazao, which is useful as a model for experimental work but is not very productive. Thus, transgenic breeding should be carried out using a hybrid silkworm strain, as usually occurs in sericulture, for silk production on a commercial scale. Therefore, there are still various issues to be resolved before it can be proclaimed that the transgenic silkworms obtained by $\mathrm{Li}$ and associates are the panacea, but it is clear that they have great potential for use in sericulture and may pave the way for further improvement and refining. Indeed, obtaining cocoons $60 \%$ bigger than normal is no trifle. King-size cocoons that would not have fit into the delicate tea cup of the princess Hsi-Ling-Shi.

\section{References}

1 Chou I. A history of Chinese entomology. Printed by Entomotaxonomia, Wugong, Shaanxi, China. 1980. VI + 215 pp. (In Chinese, with English and Esperanto summaries).

2 Goldsmith MR, Shimada T, Abe H. The genetics and genomics of the silkworm, Bombyx mori. Annu Rev Entomol 2005; 50:71-100.

3 Tomita M. Transgenic silkworms that weave recombinant proteins into silk cocoons. Biotechnol Lett 2011; 33:645654.

4 Ma L, Xu H, Zhu J, et al. Ras $1^{C A}$ overexpression in the posterior silk gland improves silk yield. Cell Res 2011; 21:934-943.

5 Grzelak K. Control of expression of silk protein genes. Comp Biochem Physiol B Biochem Mol Biol 1995; 110:671681.

6 Karnoub AE, Weinberg RA. Ras oncogenes: split personalities. Nat Rev Mol Cell Biol 2008; 9:517-531.

7 Caldwell PE, Walkiewicz M, Stern M. Ras activity in the Drosophila prothoracic gland regulates body size and developmental rate via ecdysone release. Curr Biol 2005; 15:1785-1795.

8 Tamura T, Thibert C, Royer C, et al. Germline transformation of the silkworm Bombyx mori L. using a piggyBac transposon-derived vector. Nat Biotechnol 2000; 18:81-84.

9 Tan A, Tanaka H, Tamura T, Shiotsuki T. Precocious metamorphosis in transgenic silkworms overexpressing juvenile hormone esterase. Proc Natl Acad Sci USA 2005; 102:11751-11756. 